\title{
Atuação do enfermeiro da rede primária em saúde diante do isolamento domiciliar em tempo de Covid-19
}

\author{
Primary health care nurse's performance in face of home isolation in time of Covid-19 \\ Desempeño de la enfermera de atención primaria de salud ante el aislamiento domiciliario en época \\ de Covid-19
}

\section{Ana Carolina Alvim Pessoa Alves ${ }^{1 *}$, Jéssica de Faria Barbosa ${ }^{2}$, Thauany Rodrigues Leal D’Amato ${ }^{3}$, Sebastião Jorge da Cunha Gonçalves ${ }^{4}$}

Como citar esse artigo. Alves, ACAP; Barbosa, JF; D'Amato, TRL; Gonçalves, SJC. Atuação do enfermeiro da rede primária em saúde diante do isolamento domiciliar em tempo de covid-19. Revista Pró-UniverSUS. 2020 Jul./Dez.; 11 (2): 97-101.

\section{Resumo}

A Atenção Primária à Saúde (APS), é o pilar na redução de iniquidades em saúde. Quando se fala em Pandemia, mas especificamente a da COVID-19, que atinge toda e qualquer tipo de população do mundo, é necessário que instantaneamente a associe ao cuidado primário, este que está estritamente relacionado à promoção e prevenção da saúde. Nesta perspectiva, o artigo objetivou identificar a atuação do enfermeiro na rede básica de saúde, diante as emergências em saúde pública, em tempo de Coronavírus. Para tal, realizou-se uma revisão da literatura especializada quanto as peculiaridades da APS, mudanças necessárias, como na estrutura, protocolos e fluxogramas, uso e verbas para equipamentos de proteção individual, bem como o papel do enfermeiro frente ao isolamento social. Destacou-se assim, a relevância da atenção primária como importante resposta do setor de saúde à pandemia, devendo assim ser estrutura e fortificada para tal.

Palavras-chave: APS; Enfermagem; Isolamento Social.

\begin{abstract}
Primary Health Care (PHC) is the cornerstone in reducing health inequities. When it comes to Pandemic, in particular COVID19 , which has affected each and any type of population in the world, it should instantly be associated with primary care, which is strictly concerned to the prevention and promotion of health. In this perspective, the article aims to identify the role of nurses in the basic health network, in the face of pub-lic health emergencies, in the time of Coronavírus. To this end, a specialized literature review was conducted on the peculiarities of PHC and the necessary changes in the structure, protocols, flowcharts, the uses and funds for personal protective equipment, as well as the role of nurses regards to social isolation. Thus, it is fundamental to highlight the relevance of primary health care as an important response of health sector to the pandemic, which should be structured and strengthened for this purpose.
\end{abstract}

Keywords: PHC; Nursing; Social isolation.

\section{Resumen}

La Atención Primaria de Salud (APS) es la piedra angular para reducir las inequidades en salud. Cuando se habla de Pandemia, pero en concreto de la COVID-19, que afecta a todos y cada uno de los tipos de población del mundo, es necesario asociarla instantá-neamente con la atención primaria, que está estrictamente relacionada con la promoción y prevención de la salud. En esta perspectiva, el artículo tuvo como objetivo identificar el rol del enfermero en la red básica de salud, ante emergencias de salud pública, en la época de Coronavírus. Para ello, se realizó una revisión de la literatura especializada sobre las peculiaridades de la APS, cambios necesarios, como en la estructura, protocolos y diagramas de flujo, uso y fondos para equipos de protección personal, así como el rol del enfermero ante el aislamiento social. Así, se destacó la relevancia de la atención primaria como una respuesta importante del sector salud a la pandemia, por lo que debe estructurarse y fortalecerse para ello.

Palabras clave: APS; Enfermería; Aislamiento social.

Afiliação dos autores:

${ }^{1}$ Discente do curso de Enfermagem / Universidade de Vassouras/ Vassouras/ RJ/ Brasil. ORCID: https://orcid.org/0000-0001-6854-2007 ${ }^{2}$ Discente do curso de Enfermagem / Universidade de Vassouras/ Vassouras/ RJ/ Brasil. ORCID: https://orcid.org/0000-0001-8221-0893

${ }^{3}$ Discente do curso de Enfermagem / Universidade de Vassouras/ Vassouras/ RJ/ Brasil. ORCID: https://orcid.org/0000-0003-2639-1313

${ }^{4}$ Docente do curso de Enfermagem / Universidade de Vassouras/ Vassouras/ RJ/ Brasil. ORCID: https://orcid.org/0000-0002-4228-4641. 


\section{Introdução}

Na china, em Wuhan no dia 31 de dezembro a Organização Mundial da Saúde (OMS) declarou um alerta epidemiológico de casos de pneumonia por causa desconhecida. Após estudos realizados por pesquisadores chineses descobriram uma nova cepa da família coronavírus. E no dia 11 de fevereiro de 2020 foi oficialmente intitulada Covid-19. No Brasil, o primeiro caso foi notificado em São Paulo no dia 26 de fevereiro de 2020, a partir desta data os casos, segundo o boletim do Ministério da Saúde 1 , vem crescendo. Desde então, o enfrentamento da COVID-19 mediante ao Decreto $\mathrm{n}^{\circ} 6$ de 20/03/2020, foi instituído estado de calamidade em saúde pública em âmbito nacional.

Assim, percebe-se que o novo coronavírus trata-se de uma Síndrome Gripal Aguda Grave que acomete o sistema respiratório, principalmente podendo levar a internações em âmbito de Unidade de Terapia Intensiva, sintomas leves de uma gripe ou até mesmo a não manifestação de sintomas. Nesse cenário, a transmissibilidade do SARS-COV-2 se dá pelo contato pessoaa pessoa pormeiodegotículasrespiratórias (saliva, espirro e catarro), objetos e superfícies contaminadas ou contato físico com a pessoa infectada. Sendo importante frisar sobre a efetividade da quarentena, distanciamento e isolamento domiciliar como formas de reduzir a velocidade da propagação do vírus.

Frente à assistência os diferentes níveis de atenção á saúde protagonizam o fluxo e demanda dos casos suspeitos e confirmados seja em nível hospitalar, pronto atendi-mento e atenção primária á saúde.

Atenção Primária à Saúde (APS), é o pilar na redução de iniquidades em saúde. Quando se fala em Pandemia, mas especificamente a da Covid-19, que atinge toda e qualquer tipo de população do mundo, é necessário que instantaneamente associe ao cuidado primário, que está á estritamente relacionado à promoção e prevenção da saúde. Portanto, devido à sua atuação nos territórios com alcance expressivo da população alvo, a APS deve ser estruturada e fortificada como uma importante resposta do setor de saúde à pandemia. ${ }^{2}$

Nessa perspectiva, o presente artigo tem como objetivo apresentar o papel primordial da Atenção Primária à Saúde, sendo nesse momento reorganizada para atender o curso de atendimento de casos do novo coronavírus. Sendo impactada de forma a ge-rar capacitação dos profissionais de enfermagem, coordenar equipes multidisciplinares e atender a demanda dos pacientes da micro área destinada da APS. ${ }^{3}$

\section{Materiais e Métodos}

A pesquisa tratou-se de uma revisão de literatura especializada por meio da pesquisa criteriosa de artigos científicos já existentes para expandir a compressão sobre o atual tema, visto que há um vazio literário. Para tal, utilizou-se da consulta das bases de dados Lilacs, Scielo e Google Acadêmico. As palavras de buscas utilizadas foram "Atenção Primária à Saúde", "Coronavírus", "Isolamento domiciliar" e "Enfermagem" no idioma Português, uma vez que relata a organização dos serviços de saúde frente a pandemia do novo coronavírus no Brasil. Associado a isso, recorreu-se a interpretação de guia e protocolos nacionais relacionados ao tema. Por fim, foram utilizadas literaturas do ano de 2020, em virtude de ser um assunto abordado no ano atual.

\section{Atenção Primária À Saúde Diante da Pandemia do Covid-19}

Aponta-se aqui que o impacto da pandemia do novo coronavírus atingiu não somente à parte sanitária, mas abrange as áreas econômicas, sociais, políticas e culturais. Diante do atual contexto, conduzido pelos atributos da APS (acesso, integralidade, longitudinalidade, coordenação do cuidado, orientação familiar, orientação comunitária e competência cultural), faz-se necessário apontar e identificar as orientações e adaptações a serem realizadas, lembrando-se das peculiaridades impostas pelo distanciamento social. ${ }^{3,4}$

É importante salientar que a APS deve prestar assistência à saúde tanto a população contaminada quanto a não contaminada, mas de forma que não promova o contágio. Em ambos os casos, o cuidado indicado é à distância, como por exemplo, a tele saúde que é efetiva para diminuir a disseminação do vírus e promover o cuidado contínuo e regular. Em contrapartida, a APS sempre terá a demanda de pacientes que neces-sitam do cuidado presencial, e com isso fez-se por necessário a reorganização do fluxo dos pacientes de demanda tradicional e de casos suspeitos para não ocorrer a incidência da Covid-19.

Primeiramente, as Unidades de Saúde devem se organizar para identificar os casos suspeitos e, classificar e estratificar a gravidades dos casos, sendo que quando estes forem classificados como leves o manejo clínico é de sua função, o que irá incluir a abordagem terapêutica e o isolamento domiciliar. Em casos graves, deve-se preconizar a estabilização do paciente e o encaminhamento imediato deste ao serviço de urgência. Ainda, neste cenário, é papel da unidade realizar a notificação compulsória de casos suspeitos, incluir medidas para prevenção da transmissão comunitária e ter uma vigilância ativa de novos casos. ${ }^{5}$

Isto posto, para um efetivo atendimento com segurança e qualidade na APS, é indispensável a realização de planejamentos, reorganizações dos serviços, aplicações dos recursos financeiros e estratégias de enfrentamento próprias para a defrontação 
da pandemia do SARS-COV-2.

É de grande pertinência, no momento, a reconfiguração da logística dos espaços físicos para atendimento de pacientes que demonstram sintomas de Covid-19 em locais estratégicos favorecidos de ventilação adequada e capacidade de isolamento, evitando assim fluxos cruzados com outros usuários que precisam de cuidados a nível presencial. Pensando na organização do ambiente, através de ferramentas tecnológicas, comojácitado, foipossíveldarcontinuidade nas assistências de pessoas que necessitam de cuidados regulares, monitoramento diário das condições de saúde de gestantes e pessoas vulneráveis na comunidade. ${ }^{6}$

Sendo assim, a teleconsulta de enfermagem, médica e multiprofissional está sendo relevante na redução do contato presencial entre profissionais de saúde e pacientes da Unidade Básica de Saúde (UBS). Ademais, com a elevada propagação de fakes news sobre o novo coronavírus, esse sistema de teleatendimento e mensagens em massa possibilitou ampliar a estratégia de educação em saúde desmitificando informações falsas e disseminando orientações adequadas em concordância com protocolos e embasamentos científicos.

Considerando o tempo de permanência do vírus nas superfícies, torna-se mais rigorosa e, consequentemente, mais frequente a limpeza nas Unidades Básica de Saúde (UBS), fazendo assepsia com álcool $70 \%$ nos locais e equipamentos de uso comunitário ao final de cada ciclo de atendimento a favorecer, assim, uma maior qualidade no atendimento e evitando a contaminação de usuários e profissionais.

Emse tratando de cuidados, tambémé fundamental garantir a saúde dos trabalhadores que estão atuando na linha de frente na APS certificando a disponibilidade dos equipamentos de proteção individual (EPI) e treinamento adequado para o enfrentamento da doença. Uma das mudanças obtidas por parte da gestão estratégia no atual cenário em que estamos vivenciando é a capacitação de todos os profissionais de saúde sobre protocolos e riscos ocupacionais, paramentação e desparamentação de EPI, bem como para seu adequado descarte. Neste quesito, o grande desafio é gerenciar o orçamento de compras de EPI de forma a assegurar máscaras tipo N95 ou PFF2, óculos ou face shield, luvas, gorro, capote impermeável, álcool gel $70 \%$, bem como sabão para a assistência nas unidades de saúde do Brasil. ${ }^{7}$

\section{O Enfermeiro Frente Ao Isolamento Social}

Neste momento, o acompanhamento de síndromes gripais na APS deve ser feito apenas quando os profissionais disponham de Equipamentos de Proteção Individual (EPI) adequados. Além disso, o município e as unidades devem ter estrutura e fluxos adequados para que não haja aglomeração de pacientes e que o fluxo dos casos suspeitos possa ser diferenciado dos demais, nas entradas, sala de espera, vacina e outros atendimentos.

Sendo assim, apoiar a população do território (moradores, trabalhadores, estudantes, pessoas em situação de rua ou em abrigos, dentre outros) a obter as informações atualizadas e confiáveis e a agir para que tenham as melhores condutas, bem como fornecer a atenção universal, integral e equitativa é desafio e responsabilidade da APS. Este nível de atenção não pode se furtar ao seu compromisso de ser a porta de entrada preferencial do Sistema Único de Saúde (SUS), garantindo acesso às principais necessidades de saúde da população.

Neste cenário, o enfermeiro, como gestor e integrante da equipe, deve ter atenção especial para qualidade e proteção da vida, integralidade das ações, controle social e para inclusão da educação em saúde. Sendo assim, o enfermeiro e demais profissionais que compõem a equipe, devem participar de todos os processos de trabalho no planejamento, organização, execução e avaliação das ações implantadas. ${ }^{8}$

Considerando, que desenvolvem o Serviço de Atendimento Domiciliar (SAD), prestando assistência aos pacientes com comorbidades, acamados, sem condições de saírem de seus domicílios. Neste contexto, condiz em sua maioria com doentes crônicos, em cuidados paliativos, que acabam por serem mais suscetíveis ao vírus Sars-COV-2.

É importante reforçar que a prioridade, atualmente, é ofertar a assistência por teleatendimento, até mesmo se o paciente for positivo para Sars-COV-2, caso não seja possível, recomenda-se que a equipe realize a visita domiciliar, utilizando os equipamentos de proteção individual (EPI) indicados e tomar todas as medidas de prevenção de contágio, como o distanciamento mínimo e higienização das mãos. Para realizar o acompanhamento do paciente em isolamento, é importante que os canais de contato não sejam apenas com o paciente, mas com todos da família ou mesmo de vizinhos. ${ }^{9}$

Nesse contexto, o cuidado prestado em domicilio foi remodelado de acordo com cada município e suas necessidades. Dessa forma, na assistência domiciliar, o enfermeiro previamente deve realizar uma avaliação averiguando as condições do ambiente e, se o paciente e sua família tem capacidade para tomar todas as precauções recomendadas pelo profissional, o que inclui higiene das mãos, limpeza do ambiente, higiene respiratória, questão de segurança, entre outros.

No atual momento, não há evidências científicas, normativas ou políticas diretamente relacionadas para as recomendações do cuidado de enfermagem no domicilio frente a pandemia de Covid-19, deixando assim uma lacuna no conhecimento. 9

Diante desse cenário o Ministério da Saúde desenvolveu novas cartilhas no que tange as atividades 
laborais do Agente Comunitário de Saúde e Agente de Endemias com orientações sobre as novas condutas da assistência a nível domiciliar. A partir disso, esses profissionais reorganizaram sua rotina fazendo uso de veículos digitais como televisão e redes sociais para alcançar à todos com conteúdo de caráter informativo e educacional em saúde. ${ }^{1,10}$

\section{Intersetorialidade dos Níveis de Atenção À Saúde}

A intercomunicação entre os diversos níveis de atenção à saúde gera um desafio de coordenação da ação governamental interligados com problemas multifacetados e Inter setoriais o que necessita de participação de diversos atores e organizações. No Brasil, foi materializado pelo Comitê de Crise para Supervisão e Monitoramento dos Impactos da Covid19 em 16 de Março 2020, estabelecendo a meta de organização dos fluxos para garantir a adequação das medidas necessárias do enfrentamento da Covid-19.,10

Nesse sentido, fez necessária a criação de instâncias de assistência de atendimento a pacientes suspeitos e confirmados com alternativa de não sobrecarregar a atenção primária e hospitais. Os hospitais de campanha, centro de triagem, tendas protagonizam o rastreio e tratamento de pacientes que apresentam sinais e sintomas do novo coronavírus. Além disso, as barreiras sanitárias nas divisas territoriais fiscalizam e garantem que não ocorra acesso de pessoas de outro município contaminadas ou seja que não ocorra a propagação do vírus. Outro exemplo, são as unidades básicas realizando triagem de todos que acessam a área interna da APS evitando assim a contaminação de funcionários e demais pacientes.

Ainda mais, um grande exemplo, são as Unidades Sentinelas, que são determinadas Unidades Básicas de Saúde que modificaram seu fluxo de atendimento, incluindo em massa o atendimento a pacientes suspeitos para Covid-19, de forma que ofertem o atendimento próximo das residências da população, facilitando assim o acesso ao cuidado. ${ }^{11}$ Ela funciona de maneira que o paciente, ao iniciar os sintomas gripais, procure pelo espaço, aonde será feito avaliação médica e se houver indicação, será agendada a realização do teste rápido para Covid-19, conforme a data de início dos sintomas. Dependendo do período de viremia, o teste é realizado imediatamente.

\section{Considerações finais}

Mediante o estudo e análise dos artigos para construção do mesmo, é possível observar as adversidades impostas a atenção primária à saúde no que se refere a Covid-19, assegurando os subsídios destinados a saúde e capacitaçáo da equipe multıprohissional.

Muitos são os desafios encontrados pela APS na reorganização dos serviços de saúde. Mas observase que é imprescindível que haja a intersetorialidade dos serviços, garantindo assim, investimentos, capacitações, tecnologias e reconhecendo a singularidade do trabalho executado. Com estes recursos, pôdese garantir melhoria na qualidade do serviço prestado, bem como, melhorar não apenas a técnica, mas permitir que os profissionais a executem de forma mais digna e humanística.

Os impactos atuais e futuros referentes a essa crise epidemiológica serão vividos em condições sociais, econômicas e estruturais em saúde do país que enfrentam a pandemia da Covid-19. Diversas são as lacunas na literatura acerca da mobilização dos órgãos que assistem a população em serviço de saúde, o que se sabe é que a atenção primária segue sendo o esteio em saúde garantindo o pilar da equidade, universalidade e integralidade a toda comunidade.

\section{Referências}

1. Brasil. Ministério da Saúde. Protocolo de Manejo Clínico do Corona Vírus (CO-VID-19) na Atenção Primária à Saúde. Brasília: Secretaria de Atenção Primária à Saúde, 2020. 33 p.:il. Disponível em: https://saude.gov. br/images/pdf/2020/marco/3 0/20200330-ProtocoloManejo-ver06-Final.pdf. Acesso 15 ago 2020.

2. Sarti TD, Lazarini WS, Fontenelle LF, Almeida APSC. Qual o papel da Atenção Primária à Saúde diante da pandemia provocada pela COVID19? 2020; Epidemiol. Serv. Saude, Brasília, 29(2): e2020166. Disponível em: https://www.scielosp.org/article/ress/2020.v29n2/e2020166/

3. SAPS. Secretaria de Atenção Primária à Saúde. Ministério da Saúde. Coronavírus (COVID-19). Protocolo de Manejo Clínico do COVID19 na Atenção Primária à Saúde. Março 2020. Disponível em: https:// portalarquivos2.saude.gov.br/

4. SAPS. SecretariadeAtençãoPrimáriaàSaúde(SAPS). Recomendações para ade-quação das ações dos agentes comunitários de saúde frente à atual situação epide-miológica referente ao covid-19. VERSÃO 2. Brasília- DF Março de 2020. Dispo-nível em: https://portalarquivos2.saude.gov.br/

5. Barbosa SP, Silva AVFG. A Prática da Atenção Primária à Saúde no Combate da Covid-19. APS EM REVISTA; 2(1): 17-19 | Janeiro/Abril 2020. Disponível em: ISSN 2596-3317 - DOI 10.14295/aps.v2i1.68.

6. Cabral ERM, Melo MC, Cesar ID, Oliveira REM, Bastos TF, et al. Contribuições e desafios da Atenção Primária à Saúde frente à pandemia de COVID-19. InterAm J Med Health 2020;3:e202003012.

7. Meneses AS. Gerenciamento Emergencial de Recursos da Atenção Primária a Saú-de no Enfrentamento à Pandemia da COVID-19. SciELO Preprints, 2020. DOI: https://doi.org/10.1590/SciELOPreprints.557.

8. Barra RP, Moraes EN, Jardim AA, Oliveira KK, Bonato PCR, et al. A importância da gestão correta da condição crônica na Atenção Primária à Saúde para o enfren-tamento do Covid-19 em Uberlândia, Minas Gerais. APS EM REVISTA - Artigos; 2(1): 38-43 | Janeiro/Abril - 2020 Especial COVID-19 ISSN 2596-3317 - DOI 10.14295/aps.v2i1.64.

9. Tonin L, Lacerda MR, Caceres NTDG, Hermann AP. Recomendações em tempos de COVID-19: um olhar para o cuidado domiciliar. Rev. Bras. Enferm.; Brasília 2020 Epub June; 73(Suppl 2): e20200310. Disponível em: https://www.scielo.br/scielo.php?pid=S0034$71672020001400401 \&$ script $=$ sci_arttext\&tlng=pt

10. CONASEMS. Conselho Nacional de Secretarias Municipais de Saúde - CO-NASEMS; Conselho Nacional de Secretários de Saúde - CONASS. Guia Orienta-dor para o enfrentamento da pandemia Covid-19 na Rede de 
Atenção à Saúde. Bra-sília, maio de 2020 .

11. Nedel F. Enfrentando a COVID-19: APS forte agora mais que nunca! APS EM REVISTA; 2020 abr.;2(1):11-16. Disponível em: https:// apsemrevista.org/aps/article/view/68 